\title{
The Relationship Between Follow-up Appointments and Access to Primary Care
}

\author{
Megan E. Price, MS ${ }^{7}$, Nicolae Done, $P h D^{1,2,3}$, and Steven D. Pizer, $P h D^{1,4}$ \\ ${ }^{1}$ Partnered Evidence-based Policy Resource Center, US Department of Veterans Affairs, VA Boston Healthcare System, Boston, MA, USA; ${ }^{2}$ Analysis \\ Group, Boston, USA; ${ }^{3}$ Department of Psychiatry, Boston University School of Medicine, Boston, USA; ${ }^{4}$ Department of Health Law, Policy, and \\ Management, Boston University School of Public Health, Boston, USA.
}

BACKGROUND: Health care operations managers need to balance scheduling frequent follow-ups for patients with chronic conditions and fitting in patients requiring care for new complaints.

OBJECTIVE: We quantify how frequency of follow-up visits corresponds with access to care for patients receiving care from the Department of Veterans Affairs (VA).

DESIGN: We use patient data collected between October 2013 and June 2016 by the Survey of Healthcare Experiences of Patients (SHEP). Our sample is comprised of 94,496 patients. We estimate logistic models with 1 month lagged facility-level predictors.

MAIN MEASURES: We calculate monthly measures characterizing facility-level service provision, including the average time between successive primary care visits, the average primary care visit length, the percentage of primary care appointments that are overbooked, the percent of visits that are unscheduled (i.e., walk-ins), and the ratio of patients to providers. We control for economic factors that are associated with health care supply and demand, including median household income, veteran priority status, the Zillow Housing Price Index, and veteran unemployment rates. We also control for patient demographics. PATIENTS: We restrict the data to patients with at least one in-person primary care visit who have provided information on their ability to access urgent and routine care. KEY RESULTS: We find that shorter average follow-up times are associated with better access for patients needing urgent or routine care. A 1-month increase in the average time between successive primary care visits is associated with $10 \%(p<0.001)$ lower odds of reporting being able to access urgent care within 1 day and $13 \%$ $(p<0.001)$ lower odds of reporting usually or always being able to access routine care when needed.

CONCLUSION: Facilities with higher average follow-up times are more likely to have patients report that they are unable to quickly access urgent or routine primary care.

This work was previously presented at two conferences: the Annual Conference of the American Society of Health Economists in June 2018, and the Academy Health Annual Research Meeting in June 2019.

Electronic supplementary material The online version of this article (https://doi.org/10.1007/s11606-020-05785-3) contains supplementary material, which is available to authorized users.

Received July 3, 2019

Accepted March 9, 2020

Published online March 27, 2020
KEY WORDS: access to care; wait times.

J Gen Intern Med 35(6): 1678-83

DOI: $10.1007 / \mathrm{s} 11606-020-05785-3$

(c) Society of General Internal Medicine (This is a U.S. government work and not under copyright protection in the U.S.; foreign copyright protection may apply) 2020

\section{INTRODUCTION}

Timely access to care is one of the essential features of a highquality health care system. ${ }^{1}$ Wait times in the Department of Veterans Affairs (VA) health care system are strongly associated with patient satisfaction ${ }^{2,3}$ and local market characteristics. ${ }^{4}$ However, primary care clinics balance the provision of care for many patients with a variety of medical conditions, treatment needs, and preferences. US veterans use ambulatory primary care services for a wide-ranging set of reasons, including routine care, mental health, minor but urgent health problems, and referrals to more advanced specialty services. Facilities must optimize operations to provide access for those with chronic conditions who need regular follow-up visits and patients seeking urgent care for more acute complaints. Moreover, facilities face constrained budgets and shortages of primary care providers, which hampers their ability to quickly adapt their supply to fluctuations in service demand.

In this paper, we quantify the relationship between measures of facility operations and patients' self-reported access to care in VA clinics at the national level. By combining VA facility-level data with nationally representative surveys on patient experiences and local market characteristics, we can assess how several operational metrics are associated with patient access to care. Evidence suggests that patients' satisfaction with access to care depends on whether care is needed right away or a check-up is needed instead. For example, patients' willingness to wait for more than 1 day to see their regular physician may differ based on whether they have an acute or a routine problem. ${ }^{5}$ Therefore, we use patients' selfreported access to both urgent and routine appointments to assess whether facility operations variables may have differential associations with these measures. In previous studies, several patient characteristics have also been found to be associated with patient satisfaction including demographic factors, socioeconomic status, and general health status. ${ }^{6,7}$ 
Primary care clinics have several ways of adjusting to variations in demand. First, they may schedule shorter appointments. In the VA, this strategy is restricted, as appointments typically can be scheduled for either $30 \mathrm{~min}$ (for returning patients) or $60 \mathrm{~min}$ (for new patients). Second, VA facilities often allow patients to show up for unscheduled appointments and will provide care in the event of no-shows or canceled appointments from other patients. Third, facilities differ in the extent to which they overbook appointments, thus anticipating no-shows and last-minute cancelations.

We assume that some of the differences in operations variables, such as follow-up time, appointment length, and overbooked/unscheduled appointments, are due to varying congestion and differences between facilities in prioritizing different appointment types. We account for all these factors in our analysis in order to isolate the relationships of interest. Finally, we account for several quality measures based on the HEDIS methodology, ${ }^{8}$ also calculated at the facility level.

\section{METHODS}

\section{Patient Sample}

Our sample is comprised of patients receiving primary care at one of 127 facilities in the VA who completed a questionnaire on patient experiences delivered through the Survey of Healthcare Experiences of Patients (SHEP). SHEP is an ongoing nationwide survey that seeks to obtain patient feedback on experiences with recent episodes of care in the VA and is modeled after the Consumer Assessment of Healthcare Providers and Systems (CAHPS) family of survey instruments.

The outpatient SHEP component selects a simple random sample of patients with completed appointments at VA facilities each month. We matched the last completed appointment date and location recorded by the VA to match individuals in the SHEP sample to visit month and facility where the visit occurred.

\section{Patient-Reported Access}

We obtained self-reported indicators of patient experiences with access to care from the SHEP survey. We use the responses regarding the patients' ability to obtain an appointment for urgent and routine primary care for patients who have completed an in-person visit at one of the VA facilities. Our dependent variables for urgent and routine care are aggregated facility-level survey responses.

The question for urgent care is worded as "In the last 12 months, how many days did you usually have to wait for an appointment when you needed care right away?" Response options are as follows: same day, 1 day, 2 to 3 days, 4 to 7 days, or more than 7 days. We label the "same day" response for urgent care as "could get urgent care same day," and the union of "same day" and " 1 day" responses as "could get urgent care within 1 day."

The survey question for routine care is worded as "In the past 12 months, when you made an appointment for a check- up or routine care with this provider, how often did you get an appointment as soon as you needed?" Response options are as follows: never, sometimes, usually, always. We label the "always" response as "could get routine care, always," and the union of the "always" and "usually" responses as "could get routine care, usually/always."

The survey also collects a comprehensive set of patient characteristics, including age, gender, race and ethnicity, educational attainment, and first spoken language. We control for these characteristics in all our models.

\section{Facility Operation Measures}

We used the VA Corporate Data Warehouse (CDW) to extract information on primary care appointments and visits between October 2013 and June 2016. This information included the facility and date of each appointment, as well as indicators for whether the appointment was overbooked or a patient visit was unscheduled. We calculated the monthly mean appointment length at the facility level in minutes by averaging individual appointment length. We obtained monthly mean follow-up time by averaging the time in days between a follow-up appointment and its corresponding initial visit. We also calculated the percent of overbooked appointments and unscheduled visits at the facility level during a given month.

To account for variation in the supply of services at each facility, we controlled for the ratio of full-time-equivalent primary care providers assigned to patient panels ${ }^{9}$ and patients enrolled at each facility. To mitigate the potential problem of endogeneity between patient experience with access to care and facility performance indicators, we lagged facility-level operations variables by 1 month. To assess if the relationship varied throughout the distribution, we constructed indicators of quartiles of the lagged facility-level variables and used these indicators as independent variables in the logistic models.

\section{Local Market Characteristics}

We controlled for local market characteristics that are related to veteran choice to seek VA care as opposed to community care. ${ }^{4}$ These include median household income, the Zillow Home Value Index, ${ }^{10}$ and veteran unemployment rates. To assess access to care outside the VA, we added Medicare Advantage Penetration - which signifies the density of private insurers in the area. We also controlled for the percent of veterans at the facility who pay copayments (priority status 7 and 8); copayment eligibility is determined by disability status and a means test. This variable was included both at a facility-level average to account for area economic characteristics, and at an individual level to account for individual attributes within the community.

\section{Health Care Quality Measures}

Facilities balancing the trade-off between more frequent follow-ups for existing patients and fitting in new patient and urgent care appointments must do so while trying not to 
compromise health care quality. We therefore control for a set of quality measures equivalent to the HEDIS measures used in private health plan assessments, adapted for the VA. We include a wide set of measures that capture the complexity of outpatient primary care services in a multi-faceted manner. These measures (defined formally in Appendix Table A1) include rates of guideline-concordant blood pressure and cholesterol management, and glycosylated hemoglobin (HbAlc) control for diabetic patients, as well as colonoscopy screening, influenza immunization, and pneumonia immunization rates.

\section{Regression Analyses}

We estimated mixed logistic regression models with the patientreported access indicators as dependent variables and lagged facilitylevel measures as independent variables. In each model, we controlled for patient-level characteristics, month and year fixed effects, and facility-level fixed effects. In separate models, we used lagged facility-level measures as continuous variables or categorized into quartiles, sextiles, and octiles with the lowest quantile used as the reference group (Tables A3-A5). Standard errors were clustered at the facility level. All analyses were performed in Stata 14.1. ${ }^{11}$

\section{Specification Testing}

In most economic transactions, including health care services, supply and demand are simultaneously determined. And although we control for several important patient and facility characteristics, we are unable to account for all relevant factors that may affect patient experience with access to care. To establish if such endogeneity biases our results, we tested for endogeneity of the facility operations variables by instrumenting with lagged facility operations variables and then conducting Hausman tests comparing the instrumented and non-instrumented models. The results showed that the instrumented and non-instrumented models were not statistically significantly different (results not shown).

\section{RESULTS}

\section{Sample Characteristics}

Our sample is comprised of 94,496 patients receiving care at 127 VA facilities. Sample characteristics are shown in Table 1. The mean respondent age was 65.9 years, with the majority of the respondents male and white. Most of the respondents (73\%) had some college education, and $17.3 \%$ were priority status 7 or 8 , indicating that their incomes were sufficiently high to be eligible for copays at the VA. Across all facilities, $39.9 \%$ of patients reported being able to access urgent care within 1 day, while $67.9 \%$ reported always or usually having easy access to routine care.

\section{Regression Results}

Our results suggest that facility mean follow-up time has a statistically significant negative association with access to
Table 1 Patient and Facility Characteristics for the Analytical Sample During the Study Period

\begin{tabular}{|c|c|}
\hline & $\begin{array}{l}\text { Mean (SD) or } \\
\text { percent }\end{array}$ \\
\hline \multicolumn{2}{|l|}{ Patient-level characteristics } \\
\hline Age (years) & $65.9(11.9)$ \\
\hline $20-39$ & 3.1 \\
\hline $40-59$ & 21.1 \\
\hline $60-79$ & 63.3 \\
\hline $80+$ & 12.6 \\
\hline Male & 93.4 \\
\hline Very good/excellent health & 23.6 \\
\hline \multicolumn{2}{|l|}{ Race/ethnicity } \\
\hline White & 79.8 \\
\hline Black & 14.4 \\
\hline Hispanic & 7.0 \\
\hline Other & 5.7 \\
\hline \multicolumn{2}{|l|}{ Highest education } \\
\hline High school & 8.5 \\
\hline Some college & 73.0 \\
\hline College and above & 18.5 \\
\hline English first language & 96.3 \\
\hline Priority status 7 and 8 & 17.3 \\
\hline \multicolumn{2}{|l|}{ Outcomes } \\
\hline Could get urgent care in 1 day or less & 39.9 \\
\hline Could get urgent care same day & 24.9 \\
\hline Could easily get routine care, usually/always & 67.9 \\
\hline Could easily get routine care, always & 43.8 \\
\hline Number of patients & 94,496 \\
\hline \multicolumn{2}{|l|}{ Facility-level characteristics } \\
\hline Mean appointment length (minutes) & $31.5(2.6)$ \\
\hline Clinician FTEs/1000 enrollees & $0.5(0.1)$ \\
\hline Average follow-up time (months) & $4.0(0.8)$ \\
\hline Percent visits overbooked & $11.0(7.1)$ \\
\hline Percent visits unscheduled & $31.7(15.0)$ \\
\hline Annual income $(\$ 10,000)$ & $5.6(1.1)$ \\
\hline Zillow House Price Index $(\$ 1000)$ & $194.7(118.4)$ \\
\hline Unemployment Rate $(\%)$ & $5.7(1.2)$ \\
\hline Medicare Advantage Penetration (\%) & $29.2(11.4)$ \\
\hline Priority 7 and $8(\%)$ & $24.1(6.6)$ \\
\hline Percent diabetics with controlled BP & $74.6(11.9)$ \\
\hline Percent diabetics with poorly controlled $\mathrm{HbA} 1 \mathrm{c}$ & $19.0(9.7)$ \\
\hline $\begin{array}{l}\text { Percent patients aged 50-75 with colonoscopy } \\
\text { screening }\end{array}$ & $81.9(7.2)$ \\
\hline Percent patients immunized against pneumonia & $90.8(6.2)$ \\
\hline $\begin{array}{l}\text { Percent patients aged 18-64 immunized against } \\
\text { influenza }\end{array}$ & $22.0(27.6)$ \\
\hline Percent patients aged $65+$ immunized against & $29.4(36.6)$ \\
\hline Patients per facility & $872(463)$ \\
\hline Number of facilities & 127 \\
\hline
\end{tabular}

both urgent and routine care. A 1-month increase in average follow-up time is associated with $10 \%$ lower odds of patients reporting that they could get urgent care within 1 day, and 13\% lower odds of patients reporting that they could usually or always get routine care when needed.

The secondary model, which breaks the operational variables into quartiles, shows that the effects are stronger at the higher end of the distribution (Fig. 1). Patients receiving care in facilities with mean follow-up time in the highest quartile (>4.4 months) have $16 \%$ percent lower odds of always being able to receive a routine appointment, compared with the first quartile of the distribution. For urgent care, patients receiving care in facilities in the highest quartile of the distribution have $8 \%$ lower odds of being able to get an appointment within 1 day (Fig. 1).] $\rightarrow$

The results from the logistic regression models (Table 2) show that mean facility appointment length has a small, but 


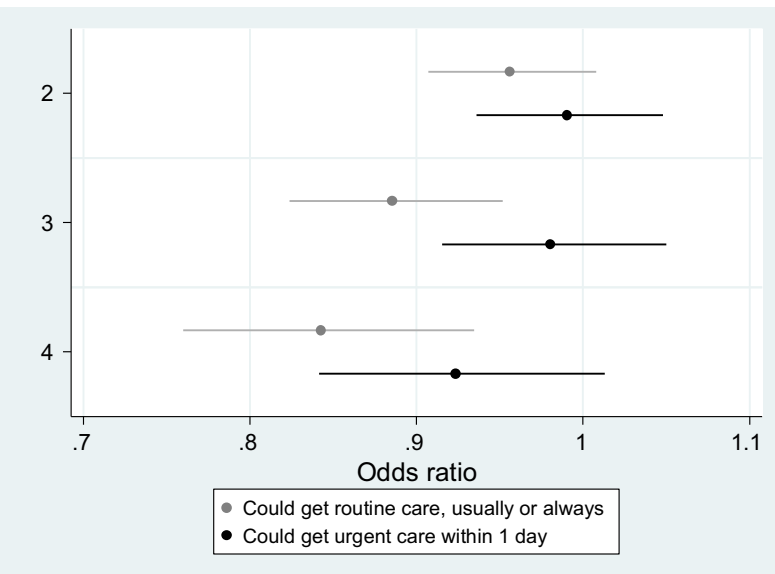

Figure 1 Secondary model: odds ratio estimates of the relationship between quartiles of lagged facility follow-up times and SHEP selfreported access to urgent and routine care, October 2013 to

June 2016. Full secondary model results are in Appendix Table A3.

statistically significant association with respondents' reported ability to access urgent appointments; longer appointments are associated with lower reported access.

Higher staffing levels are also associated with better access to care - although the results are only statistically significant for routine care. An increase of one primary care provider per 1000 enrollees is associated with $24 \%$ higher odds of a patient

Table 2 Logistic Regression Model Estimates of the Relationship Between 1-Month Lagged Facility Operations Measures, Market Characteristics, and SHEP Self-reported Access to Urgent Care, October 2013 to June 2016

\begin{tabular}{lll}
\hline \hline Variables & $\begin{array}{l}\text { (1) Could get } \\
\text { urgent care } \\
\text { within 1 day }\end{array}$ & $\begin{array}{l}\text { (2) Could get } \\
\text { routine care, } \\
\text { usually or always }\end{array}$ \\
\hline $\begin{array}{l}\text { Mean appointment } \\
\text { length, 10 min (1M lag) }\end{array}$ & $0.99 * *(-1.98)$ & $0.99(-1.51)$ \\
$\begin{array}{l}\text { Clinician FTEs/1000 } \\
\text { enrollees }\end{array}$ & $1.02(0.26)$ & $1.24 *(1.79)$ \\
$\begin{array}{l}\text { Average follow-up time, } \\
\text { month (1M lag) }\end{array}$ & $0.90 * * *(-3.24)$ & $0.87 * * *(-3.93)$ \\
$\begin{array}{l}\text { Proportion of visits } \\
\text { overbooked (1M lag) }\end{array}$ & $1.00(0.40)$ & $1.00(-0.28)$ \\
$\begin{array}{l}\text { Proportion of visits } \\
\text { unscheduled (1M lag) }\end{array}$ & $1.00(-1.26)$ & $1.00 *(-1.81)$ \\
$\begin{array}{l}\text { Local area income (1M } \\
\text { lag) }\end{array}$ & $1.19(1.36)$ & $0.96(-0.29)$ \\
$\begin{array}{l}\text { Zillow house price index } \\
\text { (1M lag) }\end{array}$ & $1.00(-0.015)$ & $1.00(-0.34)$ \\
$\begin{array}{l}\text { Local area } \\
\text { unemployment rate (1M }\end{array}$ & $1.80(0.62)$ & $1.27(0.23)$ \\
$\begin{array}{l}\text { lag) } \\
\text { Facility proportion of } \\
\text { priority 7 and 8 (1M lag) }\end{array}$ & $0.43(-0.33)$ & $0.016(-1.44)$ \\
$\begin{array}{l}\text { Area Medicare } \\
\text { Advantage penetration } \\
\text { (1M lag) }\end{array}$ & $0.37(-1.06)$ & $2.60(1.05)$ \\
$\begin{array}{l}\text { Individual priority 7 and } \\
8\end{array}$ & $1.08 * * *(3.88)$ & $1.20 * * *(8.41)$ \\
$\begin{array}{l}\text { Constant } \\
\text { Number of observations } \\
\text { Number of facilities }\end{array}$ & $\begin{array}{l}1.14(0.16) \\
94,496\end{array}$ & $\begin{array}{l}18.2 * * *(3.40) \\
94,496 \\
127\end{array}$ \\
\hline Robs Z stisting & & 127 \\
\hline
\end{tabular}

Robust Z-statistics in parentheses. $* * * p<0.01, * * p<0.05, * p<0.1$. Models also control for HEDIS quality indicators, patient age and age squared, health, patient gender, race, ethnicity, first language, education level, and facility, month, and year fixed effects. Full model results are in Appendix Table A2 reporting being usually or always able to access routine care when needed (Table 2). Overbooked and unscheduled appointments have no association with access to care (Table 2).

For both urgent and routine care, individual-level priority status 7 or 8 is strongly associated with increased patient access to care. Patients with priority status 7 or 8 have $7 \%$ higher odds of being satisfied with access to urgent care, and $20 \%$ higher odds of being satisfied with access to routine care (Table 2).

This contrasts with facility-level averages of the priority 7 and 8 population, where a higher proportion of patients in priority 7 and 8 are associated with decreased patient access to care, although the results are not statistically significant. Priority status 7 and 8 indicate that a veteran patient is expected to pay copayments due to income status - thus, this is an indication of veterans with relatively higher incomes. Area income and home prices are controlled for in this analysis - thus, this priority status indicator measures the relative income of veterans in comparison to the general population.

\section{DISCUSSION}

This study provides evidence on the relationship between measures of facility operations and access to care. We find that facilities with higher average follow-up times are more likely to have patients report that they are unable to access urgent or routine primary care. However, the associations with routine care are mostly concentrated at the higher end of the distribution, suggesting that facilities with more frequent follow-up times may be able to space patient visits further without much of an impact on patient access, while facilities with the most infrequent follow-up intervals may improve patient access by scheduling patients for more frequent return visits.

One potential explanation for the association between priority 7 and 8 veterans and higher access to care is that veterans in priority status 7 and 8 are more likely than the typical veteran to have access to a private health insurance plan, and specifically choose the VA because they are satisfied with it. In contrast, lower income veterans may not have access to other sources of health care and may continue to use the VA even if they are not satisfied.

Our study has several important limitations. First, this is an associative study. In future work, we would like to get causal estimates through instrumental variable estimation.

Second, we are not able to observe the exact reasons for which patients are scheduled for follow-up, and whether physicians are following guidelines established for conditions like diabetes or heart failure. We are assuming that some of the observed variation in follow-up time is due to differences in patient to staff ratios and differences in facility priorities between managing chronic conditions and addressing acute needs. 
Third, our measures may suffer from measurement error. The facility operations metrics are calculated at the facility level for each month and do not correspond exactly to each individual's experience. However, individual-level metrics suffer from simultaneous determination of health status and access, ${ }^{12}$ so these metrics must be constructed at the facility level. Finally, our study relies on the assumption that patients' response to the SHEP survey is dominated by their experience during the last appointment with a VA primary care provider.

The next step for future research is to extend these results to directly address how facilities can optimize operation resources in order to improve patient-reported access and care. One such step would be to review clinically appropriate follow-up times and specific clinic adherence to these follow-up times. Additional areas for future research include the relationship between patient access and clinic operations, how variations in provider workload impact the quality of care, and the relationship between telehealth and access to care.

There has been some research associating patient satisfaction to patient care and clinic operations, but more is needed. For example, there is a negative relationship between length of patient waiting times and satisfaction with access to care. ${ }^{12}$ Legler and colleagues found an association between patient satisfaction and provider's use of an integrated viewer of multiple electronic health records. ${ }^{13}$

Regarding workload, Kabdiyeva found an association between patients seen per provider and burnout. ${ }^{14}$ Based on her work, we can postulate that increasing provider workload by requiring providers to see patients more often may result in increased provider burnout.

The VA is investing heavily in telehealth, which has the potential to impact access, and thus also patient satisfaction with access. Hantke and colleagues showed that $90 \%$ of geriatric psychiatry patients who received care via video conference were at least as satisfied with their telehealth experience as with an in-person appointment. ${ }^{15}$ The VA is also expanding community care due to Mission Act requirements, ${ }^{16}$ and it is possible that pressure to change follow-up intervals would impact who is referred to community care, as a change in follow-up frequency would affect appointment demand. Feyman and Griffith showed that lower staffing levels at specialty care clinics at the VA are associated with reduced reliance on the VA and higher reliance on community care. ${ }^{17}$ We expect that this relationship may be different for primary care because veterans generally need to first be seen in primary care before they can be referred to community care. Opportunities to extend research in all of these areas will expand in the near future.

Our results, measuring the relationship between follow-up time after primary care appointments and patient-reported access, are likely to carry over into the private sector. Clinic managers may wish to increase the follow-up time to get new patients seen more quickly, but if follow-up times are too long, then patient reported with access may suffer. There is no published work on this relationship between follow-up times and patient-reported access in the private sector-thus, this study is the only available evidence. It would be valuable for a research group in a large private sector health organization to repeat this study with private data. As a VA research group, we are mostly limited to working with VA data. This may change as VA community care expands going forward.

These results could have important implications for clinic management policy. Based on patient-reported access alone, it appears that most clinics could increase follow-up times without adversely affecting patient experience. Patient experience, as measured by CAHPS scores, is a key metric for health organizations; Hospital CAHPS scores influence hospital reimbursements from CMS through their input into value-based payment metrics. ${ }^{18}$ For outpatient care, ACOs receiving CMS funds are also required to participate in CAHPS. ${ }^{19}$

Of course, our results are silent on the optimal follow-up time for chronic disease management, so individual providers will have to weigh patient access as one factor in follow-up timing. If increased follow-up time is compatible with sound medical management, it could create much needed additional space in the schedule to more promptly address concerns of new patients.

Acknowledgments: The authors wish to thank Michael Davies, MD, Mary Fields, and Manuel Alvillar for helpful comments on prior versions of this work and for logistical assistance.

Corresponding Author: Megan E. Price, MS; Partnered Evidencebased Policy Resource Center, US Department of Veterans AffairsVA Boston Healthcare System, Boston, MA, USA (e-mail: Megan. Price3@va.gov).

Funding Information This research was supported by Grant No. PEC 16-001 from the VA's Quality Enhancement Research Initiative (QUERI) and by the VA Office of Veterans' Access to Care.

\section{Compliance with Ethical Standards:}

Conflict of Interest: The authors declare that they do not have a conflict of interest.

Disclaimer: Opinions and conclusions expressed are those of the authors and do not reflect the official position of the US Department of Veterans Affairs or Boston University.

\section{REFERENCES}

1. Institute of Medicine. (2001). Crossing the Quality Chasm: A New Health System for the 21 st Century. Washington, DC: Institute of Medicine.

2. Pizer SD, Davies ML, Prentice JC. Consult Coordination Affects Patient Experience. Am J Account Care. 2017.

3. Prentice, J. C., \& Pizer, S. D. (2007). Delayed access to health care and mortality. Health Serv Res, 42(2):644-62.

4. Yee, C. A., Minegishi, T., Frakt, A. B., \& Pizer, S. D. (2019). Optimizing Resource Allocation in a Public Delivery System: Evidence from the Veterans Health Administration. Boston, MA: Partnered Evidence Based Policy Resource Center; VA Boston Healthcare System.

5. Love, M., \& Mainous, A. (1999). Commitment to a regular physician: how long will patients wait to see their own physician for acute illness? J Fam Pract, 48: 202-207. 
6. Kerr, E. A., Hays, R. D., Mitchison, A., Lee, M., \& Siu, A. L. (1999). The Influence of Gatekeeping and Utilization Review on Patient Satisfaction. J Gen Intern Med, 287-296.

7. Linn, M., Linn, B., \& Stein, S. (1982). Satisfaction with ambulatory care and compliance in older patients. Med Care, 20: 606-14.

8. Trivedi AN, Wilson IB., Charlton ME, Kizer KW. Agreement Between HEDIS Performance Assessments in the VA and Medicare Advantage: Is Quality in the Eye of the Beholder? INQUIRY. 2016.

9. Department of Veterans Affairs. (2014). VHA Handbook 1101.10 Patient Aligned Care Team (PACT) Handbook. Washington, DC: Department of Veterans Affairs.

10. Zillow Research. (2014, January 3). Zillow Home Value Index: Methodology. Retrieved from Zillow: https://www.zillow.com/research/zhvi-methodology-6032/

11. StataCorp. (2015). Stata Statistical Software: Release 14. College Station, TX

12. Prentice, J. C., Davies, M. L., \& Pizer, S. D. (2013). Which Outpatient Wait-Time Measures Are Related to Patient Satisfaction? Am J Med Qual, 227-235.

13. Legler, A., Price, M., Parikh, M., Nebeker, J., Ward, M., Wedemeyer, L. \& Pizer, S. (2019). Effect on VA Patient Satisfaction of Provider's Use of an Integrated Viewer of Multiple Electronic Health Records. J Gen Intern Med, 132-36.

14. Kabdiyeva, A. (2019, September 18). Productivity and Turnover in the Veterans Health Administration. Retrieved from U.S. Department of
Veterans Affairs: https://www.hsrd.research.va.gov/for_researchers/cyber_seminars/archives/video_archive.cfm?SessionID=3686.

15. Hantke, N., Lajoy, M., Gould, C., Magwene, E., Sordahl, J., Hirst, R., \& O'Hara, R. (2019). Patient Satisfaction with Geriatric Psychiatry Services via Video Teleconference. Am J Geriatric Psychiatry.

16. Congress of the United States of America. (2018). VA Mission Act of 2018 Washington, DC: Congress of the United States of America.

17. Feyman, Y., \& Griffith, K. (2019, September 18). The Effect of Staffing Levels on VA Reliance and Consult Wait Times. Retrieved from U.S. Department of Veterans Affairs: https://www.hsrd.research.va.gov/for researchers/cyber_seminars/archives/video_archive.cfm? SessionID=3691

18. Centers for Medicare \& Medicaid Services. (2017, December 21). HCAHPS: Patients' Perspectives of Care Survey. Retrieved from CMS. gov: https://www.cms.gov/Medicare/Quality-Initiatives-Patient-Assessment-Instruments/HospitalQualityInits/HospitalHCAHPS.html

19. Centers for Medicare and Medicaid Services. (2019, May 1). CAHPS $®$ Survey for Accountable Care Organizations Participating in Medicare Initiatives. Retrieved from CMS.gov: http://acocahps.cms.gov/

Publisher's Note: Springer Nature remains neutral with regard to jurisdictional claims in published maps and institutional affiliations. 